\title{
TECNOLOGIA DE ARMAZENAMENTO GEOLÓGICO DE DIÓXIDO DE CARBONO: PANORAMA MUNDIAL E SITUAÇÃO BRASILEIRA
}

\author{
George Câmara ${ }^{1}$ \\ ${ }^{1}$ Universidade Federal da Bahia (UFBA), Programa de Engenharia Industrial, Salvador, BA, Brasil \\ José Célio Andrade ${ }^{2}$ \\ ${ }^{2}$ Universidade Federal da Bahia (UFBA), Escola de Administração, Salvador, BA, Brasil \\ Paulo Rocha ${ }^{3}$ \\ ${ }^{3}$ Universidade Salvador - Laureate (UNIFACS), Escola de Administração, Salvador, BA, Brasil
}

\section{Resumo}

As análises cientificas e discussões quanto ao efeito das emissões antrópicas de Gases de Efeito Estufa (GEE) e suas consequências nas alterações do clima ganharam notoriedade pública nas últimas décadas. A necessidade de ações mundiais direcionadas ao combate do aquecimento global, decorrente das emissões antrópicas de GEE, tem movimentado tanto o setor público quanto o setor privado. A relação meio ambiente e economia foi um assunto discutido desde a primeira Conferência das Nações Unidas sobre o Meio Humano, realizada em Estocolmo, em 1972. Devido à relação produção/consumo/meio ambiente, o setor privado é um dos maiores interessados nas questões referentes à Governança Ambiental Global (GAG). Quanto às mudanças climáticas, embora as evidências cientificas aumentem, as respostas políticas têm tido, até agora, pouco ou nenhum impacto sobre a acumulação de emissões. A tecnologia de Captura e Armazenamento do Dióxido de Carbono ( $\mathrm{CO}_{2}$ ) em Reservatórios Geológicos (CCS) é apontada, a curto e médio prazo, como uma das principais ações de mitigação de GEE. Para o incentivo do seu uso em larga escala, estão disponiveis recursos internacionais e outros oriundos do mercado de crédito de carbono voluntário. Outro mecanismo de incentivo para o seu uso é o mercado de crédito de carbono regulado, entretanto, o CCS ainda não teve nenhuma metodologia de Mecanismos de Desenvolvimento Limpo (MDL) aprovada. O mundo já conta com diversas instituições desenvolvendo pesquisas na busca do domínio desta tecnologia. O objetivo principal deste artigo é apresentar um panorama mundial da tecnologia de armazenamento geológico do $\mathrm{CO}_{2}$ analisando a situação dessa tecnologia no Brasil. A metodologia deste artigo é composta da pesquisa exploratória, descritivo-analítica sobre o tema que privilegia estratégias e técnicas de pesquisa qualitativa e quantitativa. Para a coleta e análise dos dados secundários, foram utilizados recursos tradicionais de pesquisa. Inicialmente, foi revisada a literatura sobre a tecnologia CCS. Complementarmente, foi realizada análise de documentos institucionais, como relatórios, estudos e projetos. Os dados primários foram coletados através de consultas a especialistas. Como resultados, o artigo apresenta o posicionamento de diversos países quanto a inserção do CCS no âmbito do MDL, mais especificamente a posição do Brasil, a situação atual da estrutura de pesquisa na tecnologia CCS no Brasil, os principais motivos para investir nesta tecnologia no Brasil e por fim a identificação das lacunas existentes para o estabelecimento de um ambiente mais favorável ao domínio da tecnologia. Conclui-se que o Governo do Brasil tem estado ausente nas ações estruturantes relativas ao CCS, deixando a cargo do setor privado conduzir o desenvolvimento e domínio da tecnologia CCS.

Palavras-Chave: Armazenamento Geológico de $\mathrm{CO}_{2}$. CCS. Crédito de Carbono. MDL. 


\section{INTRODUÇÃO}

As análises científicas e discussões quanto ao efeito das emissões antrópicas de Gases de Efeito Estufa (GEE) e suas consequências nas alterações do clima ganharam notoriedade pública nas últimas décadas. Inicialmente, a atenção dada às questões ambientais tinha como foco temas envolvendo o uso dos recursos naturais disponíveis e o controle da poluição, mas tendo sempre inserido neste contexto a economia.

A relação meio ambiente e economia foi um assunto discutido desde a primeira Conferência das Nações Unidas sobre o Meio Humano, realizada em Estocolmo, em 1972. Após Estocolmo, vários passos já foram dados em direção ao estabelecimento de um sistema de governança de produção, consumo e meio ambiente mais equilibrados. Entretanto, mesmo com todo o esforço dedicado, os reflexos decorrentes das ações antrópicas no meio ambiente são latentes e cada vez mais preocupantes.

Devido à relação produção/consumo/meio ambiente, o setor privado é um dos maiores interessados nas questões referentes à Governança Ambiental Global (GAG), visto que o estabelecimento de marcos legais e infra-legais impactam diretamente nas diretrizes das empresas e seus processos produtivos, podendo comprometer sua competitividade. Segundo Porter e Brown (1996), o setor produtivo, historicamente, é visto como opositor às políticas ambientais nacionais e globais, e as questões ambientais como uma ameaça à competitividade, devido à imposição de custos adicionais. A atuação dos atores corporativos na GAG deu-se tradicionalmente de maneira indireta, utilizando a sua influência política junto aos atores estatais para vetar ou enfraquecer os regimes ambientais, com ações de lobbying.

Uma ação em parceria entre o setor público e o setor privado, que influenciou diretamente a GAG do clima, foi a bem sucedida implantação do Programa Clean Air Act's Acid Rain, do Governo Americano, no combate às chuvas ácidas naquele País. O programa foi a principal referência para a criação do mercado de créditos de carbono existente, estabelecido no Protocolo de Quioto.

Em se tratando de mudanças climáticas, numa análise mais ampla da atual situação, especificamente quanto às políticas, Helm (2008) afirma que, embora as evidências científicas da mudança climática aumentem, as respostas políticas têm tido, até agora, pouco ou nenhum impacto sobre a acumulação de emissões. Já no que diz respeito às questões tecnológicas, Pacala e Socolow (2004) afirmam que a humanidade já possui fundamentos científicos, técnicos, industriais e know-how para resolver o problema do carbono e do clima para a próxima metade do século 21. Eles apresentam as sete (07) cunhas para Mitigação das Alterações Climáticas. As cunhas estão divididas em dois grandes grupos: o primeiro, de "Redução de $\mathrm{CO}_{2}$ ou Processos Livre de Emissões de $\mathrm{CO}_{2}$ ", contendo as seguintes soluções tecnológicas: Eficiência energética; Energias Renováveis; Descarbonização dos Combustíveis Fósseis (carvão/óleo/gás); Hidrogênio e Energia Nuclear. $\mathrm{O}$ segundo grupo, com o propósito de "Capturar e Armazenar $\mathrm{CO}_{2}$ " emitido em processos industriais ou existentes na atmosfera, é composto pela Captura e Armazenamento de $\mathrm{CO}_{2}$ e a Conservação das Florestas.

Percebe-se que dentre as opções tecnológicas apontadas por Pacala e Socolow (2004) para capturar e armazenar o $\mathrm{CO}_{2}$ está o armazenamento geológico do $\mathrm{CO}_{2}$. Esta opção tecnológica é considerada crítica para a redução das emissões mundiais e tem sido amplamente pesquisada e desenvolvida por diversos países com o intuito de sua utilização em larga escala. Entende-se que o domínio desta tecnologia acarretará em vantagens competitivas para os países, dentre eles o Brasil, em função da atual Governança Ambiental Global do clima estabelecida.

Assim, este artigo tem por objetivo apresentar um panorama mundial da tecnologia de armazenamento geológico do $\mathrm{CO}_{2}$ analisando a situação dessa tecnologia no Brasil. Para atingir o objetivo proposto, fez-se uma pesquisa exploratória, descritivo-analítica sobre o tema que privilegia estratégias e técnicas de pesquisa qualitativa e quantitativa. Para a coleta e análise dos dados secundários, foram utilizados recursos tradicionais de pesquisa. Inicialmente, foi revisada a literatura sobre a tecnologia de armazenamento geológico do dióxido de carbono. Complementarmente, foi realizada análise de documentos institucionais, como relatórios, estudos e projetos. Os dados primários foram coletados através de consultas à especialistas, tais como: pesquisadores 
acadêmicos, pesquisadores de organizações não governamentais que atuam com as questões climáticas, pesquisadores de empresas e atores-chaves do Governo e organizações não governamentais como da iniciativa privada; por meio de: entrevistas com lideranças e atores-chave; observação indireta da realidade estudada através da inserção em redes de pesquisa sobre o assunto, visando à construção de espaço de cooperação de pesquisa; participação na agenda de debates e palestras sobre a temática de estudo; participação de fóruns com experts sobre o tema, como o realizado pela empresa Shell sobre o CCS; participação em eventos e feiras especializadas no tema; e observação direta, através da participação, com a chancela dos atores envolvidos, em encontros oficiais e posterior síntese dos resultados alcançados.

\section{REVISÃO DA LITERATURA}

\subsection{O armazenamento do $\mathrm{CO}_{2}$ em reservatórios geológicos}

A importância da utilização da tecnologia CGS em larga escala para a redução das emissões de $\mathrm{CO}_{2}$ é discutida, atualmente, em toda a comunidade internacional. Conforme os estudos feitos pela Internacional Energy Agency - IEA, em 2006, com perspectivas e cenários tecnológicos, a adequada captura e armazenagem de $\mathrm{CO}_{2}$ podem controlar as emissões a curto e médio prazos. O estudo afirma que, dentre as atuais tecnologias existentes ou que sejam susceptíveis a se tornarem comercialmente disponíveis nas próximas duas décadas, o CGS contribui em torno de 20 a $28 \%$ do total das reduções de emissões de $\mathrm{CO}_{2}$, tendo por base um horizonte até 2050. O G8 (grupo formado pelas oito maiores potências industriais do mundo) decidiu em junho de 2008 que apoiaria as recomendações da IEA e do Fórum de Lideranças em Sequestro de Carbono (CSFL) para o lançamento de 20 projetos de CGS em grande escala, pois o G8 entende que o CGS tem um papel crítico no combate às mudanças climáticas e aos desafios de segurança energética.

O Painel Intergovernamental para as Mudanças Climáticas - IPCC (2005) define a captura e armazenamento do $\mathrm{CO}_{2}$ em reservatórios geológicos como um processo que consiste na separação de $\mathrm{CO}_{2}$, emitido por fontes estacionárias relacionadas com a produção de energia e também de plantas industriais, no transporte deste $\mathrm{CO}_{2}$ e seu armazenamento, a longo prazo, em reservatórios geológicos, isolando-o da atmosfera. É possível separar o $\mathrm{CO}_{2}$ emitido na queima de combustíveis fósseis, processá-lo para a sua forma líquida e transportá-lo por dutos, rodovias ou por via marítima para reservatórios geológicos como minas desativadas, campos de petróleo ou outros locais onde o $\mathrm{CO}_{2}$ possa ser armazenado.

Quanto aos processos industriais, muito tem sido feito para o desenvolvimento de tecnologias para a separação do $\mathrm{CO}_{2}$ de correntes gasosas emitidas por fontes estacionárias, sendo a etapa mais onerosa e de maior consumo de energia a da captura do $\mathrm{CO}_{2}$. As tecnologias de captura do $\mathrm{CO}_{2}$ podem ser subdivididas em: de pós-combustão, de pré-combustão e combustão com oxigênio. Já quanto ao emprego da tecnologia para a captura do $\mathrm{CO}_{2}$, têm-se as tecnologias de: absorção, adorção, separação por membranas semipermeáveis, separação criogênica e o "looping químico" (Bello e Mustafa in ZILIOTTO 2010, p.223).

Com o uso das tecnologias de captura do $\mathrm{CO}_{2}$, é possível evitar a emissão deste gás na atmosfera. Mas, um dos principais pontos em discussão é o que fazer com o $\mathrm{CO}_{2}$ após sua captura. Algumas soluções tecnológicas têm ganhado força como opção para o destino do $\mathrm{CO}_{2}$, dentre elas, o armazenamento em reservatórios geológicos.

A injeção do $\mathrm{CO}_{2}$ em reservatórios geológicos é um processo que já vem sendo utilizado em alguns setores industriais. Na indústria petrolífera, existem tecnologias para a recuperação avançada de óleo ou de gás (EOR/EGR) que utilizam algumas substâncias como fluidos de injeção, dentre elas o $\mathrm{CO}_{2}$. Segundo o IPCC (2005), as principais opções para o armazenamento geológico do $\mathrm{CO}_{2}$ são: a injeção em reservatórios depletados (exauridos) de óleo e gás; o uso do $\mathrm{CO}_{2}$ para a recuperação avançada de óleo ou gás; a injeção de $\mathrm{CO}_{2}$ em reservatórios profundos saturados não usados de águas salinas; a injeção em camadas profundas de carvão mineral inexploráveis; o uso do $\mathrm{CO}_{2}$ na recuperação avançada de metano em jazidas de carvão mineral (ECBM) e, outras opções sugeridas: formações basálticas, xisto betuminoso e cavernas. 
Entende-se que o termo CCS abrange desde a captura e transporte de $\mathrm{CO}_{2}$ até o seu armazenamento geológico. Já para o termo CGS (Carbon Geological Storage), o conceito é específico para a injeção e armazenamento do $\mathrm{CO}_{2}$ em reservatórios geológicos. Além dos termos CCS e CGS, alguns autores brasileiros têm utilizado o termo CCGS no intuito de retratar desde a captura do $\mathrm{CO}_{2}$ e seu armazenamento em reservatórios geológicos (Carbon Dioxide Capture and Geological Storage).

Segundo o IPCC (2005), as principais opções para o CGS são: a injeção em reservatórios depletados (exauridos) de óleo e gás; o uso do $\mathrm{CO}_{2}$ para a recuperação avançada de óleo ou gás (EOR/EGR); a injeção de $\mathrm{CO}_{2}$ em reservatórios profundos saturados não usados de águas salinas; a injeção em camadas profundas de carvão mineral inexploráveis; o uso do $\mathrm{CO}_{2}$ na recuperação avançada de metano em jazidas de carvão mineral (ECBM) e, outras opções sugeridas: formações basálticas, xisto betuminoso e cavernas.

\subsection{Atores internacionais que atuam com o CCS}

Em função do estágio em que se encontram os projetos de CCS, as formas de incentivos e o arcabouço regulatório, alguns países estão mais avançados no domínio das tecnologias de CCS, são eles: os Estados Unidos e Canadá, assim como na União Europeia, mais especificamente a Noruega. Nos Estados Unidos, os projetos de armazenamento geológico de $\mathrm{CO}_{2}$ estão vinculados aos projetos de EOR. Para se ter uma noção melhor da quantidade de projetos existentes nos Estados Unidos, a Figura 1 apresenta as localizações destes projetos. Destaca-se que, na figura, estão representadas as localizações das fontes de emissões industriais, assim como, as fontes naturais de $\mathrm{CO}_{2}$ que servem como insumo para a EOR. Além disso, toda a rede de dutos construída para atender os projetos de EOR e também as fontes naturais de $\mathrm{CO}_{2}$ que ainda não foram exploradas.

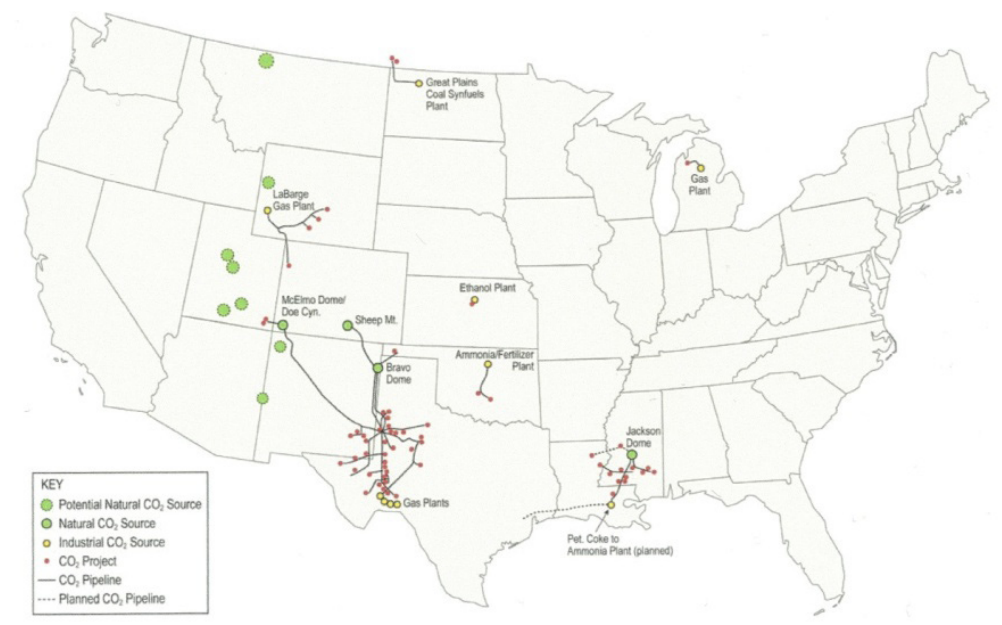

Figura 1 - Localização nos EUA dos projetos correntes de EOR com $\mathrm{CO}_{2}$ e dutovias. Fonte: National Energy Technology Laboratory (NETL, 2010).

A tecnologia de injeção de produtos em reservatórios geológicos não é muito recente. A atividade de injeção de substâncias e resíduos no subsolo dos Estados Unidos é realizada há mais de 50 anos. Esta prática é entendida como essencial para muitas atividades humanas, incluindo a produção petrolífera, a produção química, a produção alimentar, a produção industrial e mineral. De acordo com o inventário FY 2000 National Injection Well (a partir de 11/07/2006) , atualmente, existem mais de 400.000 poços com substâncias injetadas no país.

Devido à sua experiência na injeção de substâncias no subsolo, os Estados Unidos tem uma estrutura tecnológica e regulatória consolidada que favorece ao desenvolvimento de projetos de CCS. Destaca-se que esta experiência não é somente referente à injeção de $\mathrm{CO}_{2}$ e sim, também, de outras substâncias. Entretanto, este contexto favorece ao domínio tecnológico do CCS, principalmente com a experiência com a EOR da indústria de petróleo e gás. 
Digno de nota é a iniciativa entre alguns Estados do Canadá e dos Estados Unidos denominada Projeto Weyburn, iniciado em 1998. O projeto está localizado no Estado canadense de Saskatchewan. Ele utiliza o $\mathrm{CO}_{2}$ capturado de uma usina termoelétrica e injeta em campos maduros de petróleo. Mesmo tendo como objetivo inicial a EOR, após a recuperação do óleo existente será dada a continuidade da injeção do $\mathrm{CO}_{2}$ com o intuito de armazenar geologicamente o $\mathrm{CO}_{2}$ produzido pela termoelétrica. Para tanto, foi criada pelos Estados uma comissão específica para cuidar das questões regulatórias: a Interstate Oil and Gas Compact Commission (IOGCC) (ZILIOTTO 2009, p. 36) e a condução do Projeto é realizada pela Universidade de Regina (NETL, 2010).

Quanto à União Européia, existem alguns projetos em andamento, merecendo destaque o de Sleipner localizado na Noruega. Este projeto tem como objetivo armazenar $\mathrm{CO}_{2}$ em aquífero salino profundo situado no Mar do Norte e está em operação desde 1996. Além de Sleipner, outros projetos na Europa também devem ser destacados, como o de Kalundborg na Dinamarca, o de Mid-Norway também na Noruega, o de Schwarze Pumpe na Alemanha e o de Valleys no Reino Unido. Além dos já citados, outro projeto importante é o de In Salah na Argélia, já que é um dos pouco situados em um país não desenvolvido.

Para o suporte e execução dos diversos projetos de CCS existentes, o mundo tem se estruturado. Atores do setor privado, governamental e não-governamental buscam o aprofundamento e domínio das tecnologias de CCS assim como nas questões referente a financiamento e sustentabilidade destas tecnologias. Dentre as instituições existentes, destacam-se o CDP (Carbon Disclosure Project - referente à governança junto às empresas privadas), a UNFCCC (Convenção-Quadro das Nações Unidas sobre as Mudanças Climáticas), o IPCC (Intergovernmental Panel on Climate Change), a IEA (International Energy Agency) e o PNUD (Programa das Nações Unidas para o Desenvolvimento). Especificamente quanto às tecnologias do CCS, tem-se:

- Cooperative Research Centre for Greenhouse Gas (CO2CRC). Uma das principais organizações do mundo, focada na captura de dióxido de carbono e sequestro geológico. O CO2CRC é uma empresa de sociedade mista, composta por participantes do setor brasileiro e mundial, universidades e outros organismos de investigação da Austrália e Nova Zelândia, e o Commonwealth da Austrália, Estado e agências governamentais internacionais.

- Scottish Centre for Carbon Storage - University of Edinburgh and Heriot Watt University with the British Geological Survey. Centro de referência formado pela a Universidade de Edimburgo e Heriot Watt University com o British Geological Survey Edimburgo.

- Carbon Sequestration Leadership Forum (CSLF). A CSFL é uma iniciativa de âmbito ministerial internacional focada no desenvolvimento de melhores tecnologias de custo efetivo para a separação e captura do $\mathrm{CO}_{2}$, o transporte e o armazenamento. Por ser uma instituição formada por governos, sua secretaria é de responsabilidade do U.S. Department of Energy.

- Fundação Bellona. A Fundação Bellona considera a captura e armazenamento de $\mathrm{CO}_{2}$ (CCS) como uma das principais soluções para combater o aquecimento global. A Bellona foi formada como uma fundação sem fins lucrativos em junho 1986.

- Carbon Capture \& Storage Association (CCSA). A CCSA foi lançada em março de 2006 para representar os interesses dos seus membros na promoção da atividade de captura e armazenamento geológico de dióxido de carbono. Com sua base em Londres, a CCSA reúne empresas especializadas na fabricação e processamento, geração de energia, engenharia e contratação, petróleo, gás e minerais.

- Carbon Capture and Sequestration Technologies Program at MIT. Iniciado em 1989, o programa é reconhecido internacionalmente como um líder neste campo.

- The Centre for Innovation in Carbon Capture and Storage (CICCS) - University of Nottingham.

- University of Regina - mantém o International Performance Assessment Centre for the Geologic 
Storage of $\mathrm{CO}_{2}$ (IPAC- $\mathrm{CO}_{2}$ ), um programa de Pesquisa em CCS em parceria com o Governo de Saskatchewan e a Shell.

De forma geral, cabe destacar também as seguintes instituições: GASSNOVA (Companhia estatal Norueguesa para o gerenciamento e apoio dos interesses do Governo da Noruega referentes ao CCS), SINTEF (organização independente situada na Escandinávia), o Global CCS Institute, as empresas francesas

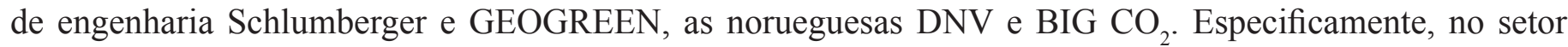
petrolífero: a Shell, a Staloil e StatoilHydro, a Chevron, a ExxonMobil, a Encanada, a BP, a Total e a Petrobras.

\subsection{Formas de incentivos internacionais para o uso do CCS}

Pode-se observar o grande potencial a ser explorado quanto à mitigação de GEE por meio da tecnologia CCS. Entretanto, são, também, de fundamental importância os impactos econômicos da inserção do CCS nos processos produtivos e o aumento de preços dos produtos em função desta inserção.

Com a percepção, por parte da economia, de que os agravos ao meio ambiente, gerados pelos processos produtivos podem ser ajustados através de instrumentos econômicos, a criação de tais instrumentos e utilização dos mesmos para a difusão e uso em larga escala de diversas tecnologias com o menor nível de emissões de GEE foi um avanço neste sentido.

Atualmente, existem dois ambientes em que são comercializados créditos de carbono oriundos da implantação e validação de tecnologias de menor emissão de GEE que são os Mercados Regulados de Crédito de Carbono, criados a partir do Protocolo de Quioto, e os Mercados Voluntários de Crédito de Carbonos. Além dos mercados de créditos de carbono, outra forma de incentivo que está sendo utilizada para financiar projetos para o armazenamento geológico de $\mathrm{CO}_{2}$ são os Fundos, principalmente os que têm a participação governamental, como é o caso do fundo criado na Noruega que obtêm os seus recursos por meio da tributação das emissões de $\mathrm{CO}_{2}$.

\subsubsection{Mercado regulado}

Desde a década de 90, o mundo está mobilizado quanto às questões das mudanças climáticas. Um reflexo disso foi a reunião realizada em junho de 1992 no Brasil - Rio de Janeiro. Esta reunião foi uma conferência internacional sobre o assunto, quando foi assinada a Convenção-Quadro das Nações Unidas sobre a Mudança do Clima - CQNUMC (United Nations Framework Convention on Climate Change - UNFCCC). Outro marco para a constituição do que hoje é denominado Regime Internacional de Mudança Climáticas foi a homologação do Protocolo de Quioto em 1997.

O Protocolo de Quioto, instrumento vinculante da Convenção, apresenta os mecanismos de flexibilização quanto às emissões de GEE. São eles: as implementações conjuntas e o comércio de emissões (Goldemberg 2003, p. 178). Os mecanismos do Protocolo de Quioto foram um agente propulsor para o estabelecimento de Mercados de Créditos de Carbono, principalmente após a sua ratificação em fevereiro de 2005. Este Mercado proposto e implementado pelo Protocolo de Quioto (chamado de compliance) tem toda a sua regulamentação estabelecida e frequentemente analisada e discutida em encontros periódicos das comissões instituídas no Protocolo e conta com a participação dos governos dos países signatários.

O mercado de crédito de carbono, criado decorrente do Protocolo de Quioto, é o mais representativo atualmente. Além disso, o preço da tonelada do $\mathrm{CO}_{2}$ comercializada nele é mais caro do que a tonelada comercializada nos Mercados Voluntários. Para ser considerada uma tecnologia que possa gerar créditos neste mercado de carbono, o CCS necessita ter sua metodologia de Mecanismos de Desenvolvimento Limpo (MDL) aprovada. 


\subsubsection{Mercado voluntário}

Um outro movimento que está sendo feito em relação ao mercado de crédito de carbono é a criação dos Mercados Voluntários. Estes mercados são formados por grupos ou setores que não tem obrigações quanto à redução de suas emissões ou por empresas situadas em países não signatários, como os Estados Unidos, que já reconhecem a necessidade de reduzir as suas emissões ou promoverem a redução de emissões em outras instituições, compensando, assim, metas internas estabelecidas.

Um exemplo é o da Bolsa do Clima de Chicago (Chicago Climate Exchange- $\mathrm{CCX}^{\circledR}$ ). A CCX ${ }^{\circledR}$ atraiu muitas empresas de grande porte, entretanto, esta iniciativa dá sinais de desgastes, já que os Estados Unidos não conta com uma meta de redução de emissões estabelecidas. Algumas iniciativas regionais e estaduais americanas como a Regional Greenhoue Gas Iniciative (RGGI) e a Western Climate Initiative (WCI) ganharam força em virtude de possíveis estabelecimentos de metas estaduais de redução de emissões.

A obtenção de créditos de carbono no mercado regulado ou no mercado voluntário, no que diz respeito à forma de agrupamento dos projetos, segue uma orientação setorial. Estes setores são previamente estudados e selecionados para que sejam elaboradas as metodologias/padrões a serem implantados e que serão passíveis de obtenção de créditos.

No mercado regulado, os setores selecionados são: indústria de energia (fontes renováveis/não renováveis); distribuição de energia; demanda de energia; indústria de transformação; indústria química, construção; transportes; mineração/produção mineral; produção de metais; emissões fugitivas de combustíveis (óleo, sólidos e gás); emissões fugitivas da produção e consumo de halocarbonos e hexafluoreto de enxofre; uso de solventes; tratamento e eliminação de resíduos; florestamento e reflorestamento e agricultura. Ao todo são quinze (15) setores que abrigam três mil trezentos e setenta e sete (3.377) projetos nas diversas metodologias aprovadas junto a United Nations Framework Convention on Climate Change (UNFCCC)2.

Quanto ao mercado voluntário, cada instituição seleciona o setor que irá trabalhar e, caso necessário, desenvolve os padrões que orientam a obtenção de créditos. Entretanto, vale ressaltar a existência da norma ANSI - GHG-PR-706, desenvolvida pela American National Standarts Institute. Esta norma foi elaborada para orientar a definição dos escopos setoriais de instituições que trabalham com validação e verificação de emissões de Gases de Efeito Estufa - GEE (ANSI, 2009).

A norma ANSI GHG-PR-706 estabelece seis (06) grandes setores onde podem ser enquadrados os projetos de obtenção de créditos de carbono, são eles: o de redução de emissões de GEE de combustíveis fósseis; o de redução de emissões de GEE de processos industriais (não-combustão, reações químicas, fugitivas e outros); uso do solo, mudanças no uso do solo e florestais; captura e armazenamento de carbono; gado e tratamento e disposição de resíduos. Estes setores são organizados em dez (10) categorias de verificação descritas da seguinte forma: geral; manufatura; geração de energia; transação de energia elétrica; mineração e produção mineral; produção de metais; produção química; extração, produção e refino de óleo e gás, incluindo petroquímicos; resíduos e agricultura, florestais e outros usos da terra.

É importante destacar que tanto os setores selecionados pela a UNFCCC e os sugeridos pela ANSI GHG-PR-706 são orientações para as instituições do mercado voluntário, pois tais instituições tem a liberdade de atuar nos diversos escopos. Sendo assim, as instituições do mercado voluntário podem estabelecer a delimitação da sua área de atuação que em certas situações pode ser determinada em função da demanda do mercado.

Tendo como exemplo a Voluntary Carbon Standard - VCS, observa-se que esta instituição segue a estrutura dos grupos de projetos e escopos estabelecidos na ANSI, desenvolve as suas metodologias/padrões e aceita as metodologias/padrões aprovados no âmbito da UNFCCC, tanto para MDLs como para JIs, e pela Climate Action Reserve. 
A instituição Gold Standart (GS) tem como referência os escopos definidos na UNFCCC assim como as metodologias aprovadas no âmbito dos MDLs, além de desenvolver as suas próprias metoologias. Outra instiruição que também adota as metodologias da UNFCC no âmbito do MDL é a American Carbon Registry - ACR. A ACR também desenvolve as suas próprias metodologias para a obtenção de créditos de carbono em outros escopos que não os do MDL, como as Reduções de Emissões por Desmatamentos e Degradação (REDD) e CCS.

Quanto a ACR é importante destacar que nesta instituição já foram emitidos créditos de carbono oriundos de projetos de CCS. Pode-se verificar três projetos, todos localizados nos Estados Unidos e feitos para empresas petrolíferas com a aplicação da tecnologia para a Recuperação Avançada de Petróleo (EOR). Outra instiuição que também se demostrou aberta a emitir creditos de carbono oriundos de projetos de CCS é a VCS, entretanto, ela ainda não foi demandada para a certificação de projetos de CCS e consequentemente não desenvolveu metodologias específicas para esta tecnologia de mitigação de GEE.

\subsubsection{Fundos}

Atualmente, a principal forma de incentivo internacional para o uso das tecnologias de CCS são os Fundos. Segundo o relatório o WorleyParsons Services Pty Ltd produzido para o Global CCS Institute em 2009, apenas no banco de dados do Global CCS Institute existem 449 projetos no mundo que tenha envolvida alguma tecnologia de CCS. Em uma observação mais detalhada, pode-se verificar a permanência de 275 projetos no mundo em diversos estágios de aplicação que estão distribuídos da seguinte forma: 34 concluídos; 26 cancelados ou atrasados; 2 retidos e 213 ativos ou previstos, sendo $101 \mathrm{em}$ escala comercial, e 62 integrados (demonstram toda a cadeia produtiva $\mathrm{CCS}$ de $\mathrm{CO}_{2}$ captura, transporte e armazenamento), dos quais 07 estão em operação.

Os 213 projetos em curso ou planjeados foram, então, analisados ainda pela sua dimensão e tipo de projeto de CCS. Dos 213 projetos ativos ou previstos, 101 são de escala comercial. Destes, 62 projetos são considerados como integrados, ou seja, eles demonstram toda a cadeia produtiva $\mathrm{CCS}$ de $\mathrm{CO}_{2}$ captura, transporte e armazenamento. Sete deles já estão em operação. Isso deixa 55 projetos que potencialmente poderiam ser candidatos ao objetivo do G8 (WORLEYPARSONS 2009, p.20).

No que diz respeito à origem dos recursos para os projetos de CCS, grande parte é oriunda de medidas de financiamento dos governos e outras agências, principalmente para os projetos em escala piloto. Atualmente, estima-se que estejam disponíveis, aproximadamente, entre U\$ 17 a U\$ 20 bilhões de recursos para projetos de CCS (WORLEYPARSONS 2009, p.41).

\section{RESULTADOS E DISCUSSÕES}

\subsection{Posição do Brasil no panorama mundial na inclusão do CCS no MDL}

O principal ambiente regulatório internacional onde os países e demais atores são solicitados a emitirem suas opiniões é a UNFCCC, haja vista que para se tornar uma tecnologia reconhecida e elegível no âmbito dos MDL as tecnologias são submetidas ao crivo das delegações e também são feitas consultas aos Governos e aos demais atores envolvidos, como centros de pesquisas, fundações, organizações não-governamentais, dentre outros.

A possibilidade de inclusão de projetos de CCS como MDL foi discutida, inicialmente, na primeira sessão da 11 a Conferência das Partes da Convenção-Quadro das Nações Unidas sobre Mudança do Clima (COP 11) e na $1^{\text {a }}$ Reunião das Partes do Protocolo de Quioto (MOP 1) em Montreal-Canadá em 2005. Em dezembro de 2006, em Nairóbi, foi decidido que seria necessário mais tempo para considerar as questões referentes à adoção de uma metodologia do CCS como MDL. Na terceira reunião em dezembro de 2007, decidiu-se postergar a decisão para a reunião de 2008 em Polznan na Polônia. Em Polznan, nenhuma decisão relevante foi adotada e as definições foram novamente postergadas para Copenhague na Dinamarca, quando o CCS foi reconhecido como uma tecnologia de redução possível. 
Em paralelo às discussões das reuniões, a UNFCCC solicitou a opinião de diversos Governos e outros atores como fundações e ONG's quanto à tecnologia CCS como MDL. A Figura 3 apresenta de forma sintetizada a posição dos Governos e Atores consultados pela UNFCCC até 2010 quanto à inclusão do CCS como um MDL.

\begin{tabular}{|c|c|c|c|c|c|c|c|c|c|}
\hline \multirow{2}{*}{ País } & \multicolumn{4}{|c|}{ Posição em } & \multirow{2}{*}{ Instituições e ONG's } & \multicolumn{4}{|c|}{ Posição em } \\
\hline & 2010 & 2009 & 2008 & 2007 & & 2010 & 2009 & 2008 & $\begin{array}{l}2007 \\
2006 \\
\end{array}$ \\
\hline Brasil & & & & & Bellona & & & & \\
\hline Austrália & & & & & Carbon Capture \& Storage Association & & & & \\
\hline Indonésia & & & & & South African Centre for CCS & & & & \\
\hline Noruega & & & & & Carbon Sequestration Leadership Forum & & & & \\
\hline Espanha pela União Europeia & & & & & Eskon & & & & \\
\hline Venezuela & & & & & Eureletric & & & & \\
\hline Nova Zelândia & & & & & Greenpeace & & & & \\
\hline Arábia Saudita & & & & & International Chamber of Commerce & & & & \\
\hline Slovênia & & & & & International Emissions Trading Association & & & & \\
\hline Portugal pela União Europeia & & & & & World Coal Institute & & & & \\
\hline Canadá & & & & & Indian Institute of Technology Kharagpur & & & & \\
\hline Coreia & & & & & Indian Institute of Management Indore & & & & \\
\hline \multirow{2}{*}{\multicolumn{5}{|c|}{ Contra as tecnologias de CCS }} & $\begin{array}{l}\text { International Petroleum Industry } \\
\text { Environmental Conservation Association } \\
\text { WWF }\end{array}$ & & & & \\
\hline & & & & & International Risk Governance Council & & & & \\
\hline \multirow{2}{*}{\multicolumn{5}{|c|}{$\begin{array}{l}\text { Aceita as tecnologias de CCS e contra a inclusão do CCS como MDL } \\
\text { A favor da inclusão das tecnologias de CCS como MDL }\end{array}$}} & Pembina & & & & \\
\hline & & & & & Sustain US & & & & \\
\hline \multicolumn{5}{|c|}{ Extremamente a favor das tecnologias de CCS e sua inclusão como MDL } & $\begin{array}{l}\text { The Norwegian Forum for Environment and } \\
\text { Develonment }\end{array}$ & & & & \\
\hline
\end{tabular}

Figura 3 - Posicionamento dos Países e demais Atores quanto a inclusão das tecnologias de CCS como MDL.

Fonte: Elaboração própria.

Observa-se na Figura 3 que, dos países consultados pela UNFCCC quanto à inclusão do CCS no âmbito do MDL, somente o Brasil e Portugal em 2007, representando a União Europeia, foram contrários. Destaca-se que a posição da União Europeia muda em 2010, quando a mesma foi representada pela Espanha, entretanto, a posição do Brasil se mantém contrária à inclusão do CCS no âmbito do MDL nos dois posicionamentos, tanto em 2008 quanto em 2010. No que tange às demais instituições e ONG's, somente o Greenpeace foi contra ao CCS, entretanto, existe uma quantidade maior de atores que são contra a inclusão do CCS no âmbito do MDL.

Na COP 16, realizada em dezembro de 2010 em Cancún no México, o CCS foi reconhecido como uma tecnologia importante para o combate às emissões de GEE e considerado elegível no âmbito dos MDL's. Entretanto, alguns passos ainda precisam ser dados em 2011 para que seja validada a adoção do CCS como um MDL. Destaca-se que para se tornar elegível o CCS teve que ser aceito no Executive Board (EB), que conta com a participação de diversos países. O Brasil esteve presente nesta reunião na qual foi aprovada a elegibilidade do CCS no MDL, mesmo sendo um dos países que é contra a inclusão do CCS no âmbito do MDL, posição esta externada antes da COP 16 em documento enviado àUNFCCC pelo Governo do Brasil.

O Governo do Brasil se pronunciou duas vezes junto à UNFCCC quanto à adoção do CCS como MDL. A primeira vez em 2007 e mais atualmente em 2010, antes da realização da COP16. De forma geral, o Brasil não é contra o uso do CCS, contudo, entende que estas tecnologias não são elegíveis como projetos de MDL por diversas razões, dentre as quais destacam-se as seguintes:

O Brasil apoia a aceleração da pesquisa sobre tecnologias de CCS e apoia o desenvolvimento, implantação e difusão, incluindo a transferência dessas tecnologias CCS que já estão, pelo 
menos, na fase de demonstração, sob análise da UNFCCC [...]. O Brasil está consciente de que a aplicação da CCS nos países em desenvolvimento dependerá da maturidade técnica, custos de difusão e transferência de tecnologia e avaliação das questões ambientais, tendo em conta que este processo é intensivo em capital e tecnologia (UNFCCC, 2010).

E, especificamente, quanto à adoção das tecnologias de CCS como um MDL:

Levando em consideração as modalidades e procedimentos dos MDL, tais tecnologias têm implicações e características que são incompatíveis com a natureza e as características das atividades de projeto MDL. Questões como o fugas, limite do projeto, a responsabilidade a longo prazo e permanência tem muitas implicações adicionais. Algumas destas questões foram analisadas por instituições confiáveis, mas nenhuma solução satisfatória foi alcançada, especialmente se levando em conta as características das atividades de um projeto de MDL. Algumas outras questões importantes a respeito da natureza dos MDL e possíveis impactos econômicos e de mercado ainda não foram avaliados (UNFCCC, 2010).

O Brasil acredita que os incentivos do MDL devem ser bastante utilizados para promover tecnologias mais limpas e renováveis, que apontam claramente para a descarbonização dos padrões atuais de produção e consumo e não para promoção do uso do petróleo, gás e carvão mineral. Projetos de CCS, nos países em desenvolvimento, poderiam acontecer num outro contexto, com mecanismos financeiros específicos, financiamentos e parcerias no âmbito da UNFCCC, mas não como um mecanismo de compensação, gerando créditos de carbono a ser usados por países do Anexo I, como acontece com o MDL. A inclusão de projetos de CCS dentro do MDL pode diminuir a ênfase na busca de outros mecanismos financeiros mais adequados no âmbito da UNFCCC ou das políticas governamentais (UNFCCC, 2010).

Os riscos inerentes às novas tecnologias de CCS só seriam minimizados com a consideração da possível aplicação comercial de tecnologias maduras de CCS. O único mercado maduro de tecnologias para o armazenamento geológico de $\mathrm{CO}_{2}$ listado no relatório do IPCC é a Recuperação Avançada de Petróleo (EOR). O Governo do Brasil acredita que o MDL não foi concebido a fim de subsidiar a produção de petróleo e gás natural dos países com produção terrestre que tem custos muito baixos de produção. Este tipo de projeto não pode depender ou ser complementado por incentivos oriundos do MDL. Os produtores de combustíveis fósseis não precisam deste tipo de subsídio, tendo em conta que o preço atual do petróleo é superior a U\$ 80/bbl. Além disso, essas empresas detêm know-how e investimentos significativos na área das tecnologias de CCS. O MDL também não deve ser destinado para incentivar a extração de metano em minas de carvão (UNFCCC, 2010).

Para o Governo do Brasil, uma das principais características das atividades do projeto de MDL é a geração de créditos a curto prazo, entretanto seus benefícios devem ser reais e mensuráveis a longo prazo. Projetos de CCS no âmbito dos MDL poderiam gerar uma quantidade importante de créditos a curto prazo, mas não trariam benefícios a longo prazo. A consequência seria o ganho de tempo para a economia atual, baseada em combustíveis fósseis. A inclusão de projetos de CCS no MDL poderia gerar um grande número de projetos, especialmente concentrados em poucos países. Isso impediria a participação mais equitativa dos países que não fazem parte do Anexo I no âmbito do MDL e, certamente, iria criar obstáculos adicionais para projetos de pequena escala. Além disso, adiaria investimentos importantes que poderiam levar à introdução de tecnologias de energia renovável no mundo em desenvolvimento. A este respeito, o CCS é claramente uma tecnologia que pode ser usada por países do Anexo I nos seus esforços de mitigação, já que eles têm uma enorme infra-estrutura voltada para o uso de combustíveis fósseis e a necessidade de reduzir significativamente suas emissões no curto prazo (UNFCCC, 2010).

O CCS é tipicamente uma tecnologia de transição para ser usada na passagem de uma economia baseada no uso de combustíveis fósseis para uma economia de baixa intensidade de carbono. O Governo do Brasil reconhece que o CCS pode ser útil durante um longo tempo, antes que o mundo possa confiar 
plenamente nas energias renováveis para atender suas necessidades energéticas. Sendo assim, o CCS poderia ser uma das tecnologias-ponte até que os países possam ter plena confiança nas energias renováveis, mas, ao mesmo tempo, o CCS, no âmbito do MDL, acarretaria em incentivos perversos para o aumento da produção de energia fóssil nos países em desenvolvimento, o que permitiria reforçar a lacuna tecnológica existente entre o mundo desenvolvido e o mundo em desenvolvimento (UNFCCC, 2010).

\subsection{Potencial de uso do CCS no Brasil}

Em 29 de dezembro de 2009, após a realização da COP 15, o Governo do Brasil aprovou a Lei da Política Nacional de Mudança Climática. Segundo a lei aprovada, o Brasil "adotará, como compromisso nacional voluntário, ações de mitigação das emissões de gases de efeito estufa, com vistas em reduzir entre $36,1 \%$ (trinta e seis inteiros e um décimo por cento) e 38,9\% (trinta e oito inteiros e nove décimos por cento) suas emissões projetadas até 2020" (BRASIL, 2009, p. 5).

Outro fator que potencializa o uso da tecnologia do CGS no Brasil é a ampliação da participação na geração de energia elétrica por meio de usinas termoelétricas. Mesmo o Brasil tendo uma matriz energética baseada na produção de energia por meio de usinas hidroelétricas, o Plano Nacional de Energia 2030, publicado em 2007 (BRASIL, 2007), aponta a necessidade de expansão de usinas termoelétricas à base de carvão mineral, nuclear, biomassa e gás natural. A necessidade de crescimento da geração de energia por usinas térmicas seria da seguinte ordem, num horizonte entre os anos de 2005 a 2030 e por percentual de participação na produção:

- carvão mineral - aumento de 1,3\% (passando de 1,7\% em 2005 para 3\% em 2030);

- nuclear - aumento de 2,2 \% (passando de 2,7\% em 2005 para 4,9\% em 2030);

- biomassa - aumento de 3,2\% (passando de $0 \%$ em 2005 para 3,2\% em 2030); e,

- gás natural - aumento de 4,9\% (passando de 3,8\% em 2005 para 8,7\% em 2030).

Com a ampliação da geração de energia por meio de usinas termoelétricas, a projeção para a produção de energia elétrica por meio de usinas hidroelétricas é de redução de $12,1 \%$, passando de uma participação de 89,5\% em 2005 para 77,4\% em 2030. Caso as projeções se consolidem, as emissões de GEE deverão ser ampliadas ao longo dos 25 anos do estudo feito pelo Plano Nacional de Energia, o que significaria o alcance de mais de $770 \mathrm{Mt} / \mathrm{CO}_{2}$ em 2030, com taxa de crescimento anual de 3,5\% sobre 2005 (BRASIL, 2007).

De forma geral, se existir um incentivo para a utilização da tecnologia de CCS no Brasil, seja por meio de financiamentos públicos ou taxas sobre as emissões, estudos exploratórios apontam para um potencial de mitigação de $\mathrm{CO}_{2}$, somente para as províncias petrolíferas do Pré-sal e da Bacia do Recôncavo, estimado

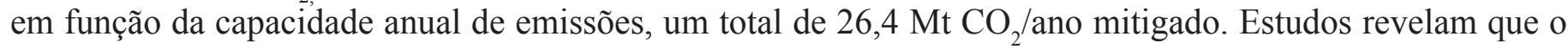
potencial dos possíveis reservatórios geológicos do Brasil de armazenamento é de aproximadamente 2000 Gt (bilhões de toneladas) de $\mathrm{CO}_{2}$ (MACHADO et al., 2009). Somente a Bacia do Recôncavo, segundo Costa apud Rocha (in ZILIOTTO 2009, p. 339), indica um consumo diário de 14,5 kt $\mathrm{CO}_{2} /$ dia.

A meta de redução das emissões brasileiras foi apresentada pelo Governo do Brasil na COP15 referente ao preenchimento do apêndice II do Acordo de Copenhague, que lista as "Ações de mitigação nacionalmente apropriadas de países em desenvolvimento". A meta proposta teve como base o ano de 2020, para quando se projetou um total de emissões de $2.703 \mathrm{Mt} \mathrm{CO}_{2}$-eq. As ações a serem tomadas para o alcance da meta estão mais detalhadas nas Ações de Mitigação Nacionalmente Adequadas (NAMAs) (IPEA 2010, p.11).

Dentre as ações previstas pelo Governo, não se faz referência ao uso do Armazenamento do $\mathrm{CO}_{2} \mathrm{em}$ Reservatórios Geológicos. Conforme o exposto anteriormente, estudos exploratórios indicam que, somente utilizando o CGS nestas duas províncias petrolíferas, a capacidade de mitigação das emissões de $\mathrm{CO}_{2}$ por ano, projetada para 2020, dessa tecnologia, seria em torno de 26,4 $\mathrm{Mt} \mathrm{CO}_{2}$ /ano mitigado, maior do que as previstas para diversas ações de mitigação. 


\subsection{Principais atores brasileiros que atuam com o CCS}

As atividades de pesquisa e desenvolvimento das tecnologias de CCS no Brasil tiveram seu começo na indústria petrolífera, mais especificamente na Petrobras. Segundo Lino (2005), testes de injeção de $\mathrm{CO}_{2}$ em campos da Bacia do Recôncavo/BA foram iniciados desde maio de 1991 no campo de Buracica. Outro setor produtivo brasileiro que também desenvolve atividades de pesquisa e desenvolvimento das tecnologias de CCS é o da indústria de exploração de carvão mineral. As pesquisas neste setor basicamente buscam o estudo da utilização convencional e não-convencional de energia fornecendo usos do carvão, tais como a recuperação avançada de metano em camada de carvão (ECBM), o metano contido em camada de carvão (CBM) e hidrogênio derivado de camada de carvão via gaseificação de carvão in situ (UCG).

Duas instituições se destacam na pesquisa e desenvolvimento das tecnologias de CCS, são elas o Centro de Pesquisa e Desenvolvimento Leopoldo Américo Miguez de Melo/Petrobras (CENPES) e o Centro de Excelência em Pesquisa sobre Armazenamento de Carbono/PUC-RS (CEPAC). No que tange à captura do $\mathrm{CO}_{2}$ emitido por fontes estacionárias, vale destacar também as atividades de pesquisas realizadas pela Universidade de Salvador (UNIFACS).

Outras instituições que apoiam a pesquisa e o desenvolvimento das tecnologias de CCS no Brasil merecem destaque, são elas: a Associação Brasileira do Carvão Mineral, a Associação Beneficente da Indústria Carbonífera de Santa Catarina, COPELMI Minerações Ltda, Rede Carvão, Instituto Ecoar, Instituto Ecoclima, dentre outras.

Devido à relação das tecnologias de armazenamento geológico de $\mathrm{CO}_{2}$ com a indústria de energia, mais especificamente a indústria petrolífera e carbonífera, outros setores, como o petroquímico e o de produção de cimento, não despertaram para o uso dessas tecnologias como uma ação de mitigação dos GEE emitidos. Isso seria de grande importância para o domínio e difusão das mesmas no país.

Numa perspectiva mais abrangente dos diversos temas correlatos, destacam-se como principais centros nacionais de pesquisas os seguintes:

- Mudanças Climáticas: o Instituto Nacional de Pesquisas Espaciais (INPE);

- MDL e Governança Ambiental Global: grupo de pesquisa do Laboratório de Análise Política Mundial (LABMUNDO) criado em 2007 no Núcleo de Pós-Graduação em Administração (NPGA) da Universidade Federal da Bahia (UFBA).

Dentre as instituições privadas, destaca-se o CEBDS (Centro Empresarial Brasileiro para o Desenvolvimento Sustentável), especificamente a Câmara Temática de Energia e Mudança do Clima. No âmbito público, tem-se como principal ator institucional o Ministério da Ciência e Tecnologia (MCT) já que é o responsável por conduzir as ações para a obtenção de créditos de carbono dos projetos realizados por organizações brasileiras junto à UNFCCC.

No contexto dos atores que estão diretamente relacionados com as tecnologias de CCS no Brasil, cabe um destaque ao trabalho que vem sendo feito pela Petrobras. Conforme o Inventário das Emissões de Gases de Efeito Estufa - ano base 2008, elaborado pela Petrobras e apresentado ao Programa Brasileiro GHG Protocol, as emissões da Petrobras passaram de 20,7 $\mathrm{MtCO}_{2}$ em 1990 para 50,8 $\mathrm{MtCO}_{2}$ 2008. Neste mesmo relatório, a empresa informa que, dentre as mensagens principais de combate as emissões, a empresa "Investe agressivamente em Programas de P\&D para viabilizar novas tecnologias, incluindo Renováveis, Eficiência Energética e CCGS (captura, sequestro, transporte, armazenamento geológico e monitoramento do $\mathrm{CO}_{2}$ ), envolvendo diversas universidades e institutos de pesquisa" (PETROBRAS, 2009).

Além de toda a atenção destinada pela Petrobras às emissões oriundas dos processos de exploração, produção, transporte, refino e distribuição da cadeia da indústria petrolífera, outro fator extremamente relevante está sendo objeto de estudo pela empresa, a alta presença de $\mathrm{CO}_{2}$ encontrada no hidrocarboneto do Pré-sal. 
De forma geral, as características do hidrocarboneto encontrado nos campos do Cluster do Pré-sal são as seguintes: grau API alto, entre 28 - 30 ${ }^{\circ}$; alta relação de solução de gás, entre $200-300 \mathrm{~m} 3 / \mathrm{m} 3$ e presença de $\mathrm{CO}_{2}$ no hidrocarboneto, entre 8 a 12\% (ALMEIDA et al., 2010). O percentual de 8 a $12 \%$ de $\mathrm{CO}_{2}$ presente é considerado significativo em comparação com a composição de outros hidrocarbonetos.

Diante desta realidade, a Petrobras tem estudado opções para tornar a exploração e produção do hidrocarboneto encontrado no Pré-sal viável quanto à perspectiva da sustentabilidade. Segundo Almeida et al. (2010), as seguintes opções estão sendo avaliadas tecnicamente e economicamente para o aproveitamento e uso do $\mathrm{CO}_{2}$ contido no hidrocarboneto do Pré-sal: EOR nos reservatórios do Pré-sal; armazenamento de $\mathrm{CO}_{2}$ em aquíferos salinos; EOR em campos de óleo pesado na Bacia de Santos; o armazenamento de $\mathrm{CO}_{2}$ em campos de gás exauridos; o armazenamento de $\mathrm{CO}_{2}$ em cavernas de sal a serem construídas na área do cluster; transporte de $\mathrm{CO}_{2}$ para o continente e comercialização de plantas industriais (opção de uso/armazenamento não geológico).

Apesar de todas as alternativas serem igualmente analisadas, a opção preferida para o escoamento do $\mathrm{CO}_{2}$ presente no hidrocarboneto do Pré-sal parece ser a reinjeção nos reservatórios do próprio Pré-sal, pois pode-se obter um benefício duplo nesta estratégia: o aumento no fator de recuperação do hidrocarboneto e a verificação do efetivo armazenamento do $\mathrm{CO}_{2}$ produzido (ALMEIDA et al., 2010).

Com o intuito de se antecipar aos problemas de âmbito ambiental e também para influenciar a Governança Ambiental Global (GAG) do clima, a Petrobras vem investindo intensamente no domínio da tecnologia de Armazenamento em Reservatórios Geológicos de $\mathrm{CO}_{2}$. Um dos principais resultados destes investimentos é o centro de pesquisa e desenvolvimento da Petrobras, o CENPES, e os programas Proclima e PRO- $\mathrm{CO}_{2}$, nos quais são investidos anualmente \$ 25 milhões. Além do CENPES e dos programas, a Petrobras estabeleceu uma Rede Nacional de Tecnologia em Mudanças Climáticas e Captura e Sequestro de $\mathrm{CO}_{2}$. Esta rede envolve 13 instituições e entre 2006 a 2009 foram investidos \$ 30 milhões em projetos (ALMEIDA et al., 2010).

\section{CONSIDERAÇÕES FINAIS}

As tecnologias de captura e armazenamento de $\mathrm{CO}_{2}$ em reservatórios geológicos têm ganhado notoriedade internacional pelo seu alto potencial para mitigar as emissões oriundas de fontes estacionárias. Estas tecnologias estão sendo pesquisadas e desenvolvidas numa velocidade muito grande nos países desenvolvidos. O conhecimento e domínio destas tecnologias é um importante passo para um país se posicionar e se fazer ouvir enquanto ator nos principais fóruns de discussão do tema Mudanças Climáticas. A evidência da corrida para o domínio das tecnologias de CCS pode ser refletida na quantidade de projetos, de recursos disponíveis e mecanismos de fomento.

O Governo do Brasil não tem apoiado de forma contundente a pesquisa e o desenvolvimento das tecnologias de CCS, deixando a cargo da iniciativa privada e tendo como justificativa a sua falta apoio na estreita relação destas tecnologias com o setor energético. Por outro lado, a iniciativa privada no Brasil só conta com os investimentos da Petrobras e da indústria de exploração de carvão mineral. É importante ressaltar que, pelo fato do setor energético ser estratégico para o país, o desenvolvimento de um ambiente favorável para o domínio das tecnologias do CCS é de extrema relevância. Mesmo que num futuro conclua-se que estas tecnologias não são tão promissoras quanto tem se apresentado.

A ausência da participação governamental, num momento em que a comunidade científica internacional assim como setores produtivos importantes e Governos de outros países se mobilizam na busca do domínio das tecnologias do CCS, é no mínimo preocupante. Aspectos importantes para que se possa estabelecer um ambiente seguro para a pesquisa e desenvolvimento das tecnologias de CCS no Brasil deveriam ser discutidos, como a estrutura regulatória para estas novas tecnologias, suas formas de incentivos, definição de atores governamentais, seleção de setores industriais prioritários para a pesquisa e desenvolvimento das tecnologias 
de CCS, estabelecimento de redes de pesquisas, dentre outros.

Em virtude do exposto neste artigo, percebe-se a existência de alguns pontos relacionados com a tecnologia de CCS e sua utilização em larga escala no Brasil a serem futuramente pesquisados, dentre eles destacam-se: a percepção pública quanto à utilização das tecnologias do CCS; identificação das lacunas regulatórias existentes; estado da arte da tecnologia no Brasil e o levantamento do interesse do Governo e do setor privado.

\section{AGRADECIMENTOS}

Coordenação de Aperfeiçoamento de Pessoal de Nível Superior (CAPES).

\section{REFERENCIAS}

ALMEIDA, A. S. et al. A study on the potential for CCGS in the Pre-salt cluster of Santos Basin: the Tupi pilot application. In: Rio Oil \& Gas Expo and Conference 2010. Copyright 2010, Rio de Janeiro. Anais... Rio de Janeiro: Instituto Brasileiro de Petróleo, Gás e Biocombustíveis - IBP, Setembro de 2010.

ANSI - AMERICAN NATIONAL STANDARTS INSTITUTE. ANSI GHG Validation and Verification Body Accreditation Scoping Policy, GHG-PR-706, revision 0. 2009.

Bello, S., Mustafa, G., 2009. Separação e Captura de $\mathbf{C O}_{2}$ de Fontes Estacionárias. In: Ziliotto, M.A.. (Org.). Mudanças Climáticas, Sequestro e Mercado de Carbono no Brasil. 1 ed. Paraná: Instituto Ecoclima/UFPR, 2009, v.1. p. 219-236.

BRASIL, Ministério de Minas e Energia. Plano Nacional de Energia 2030 / Ministério de Minas e Energia; colaboração Empresa de Pesquisa Energética. Brasília: MME: EPE, 2007. p. 324 : il.

BRASIL. Lei n ${ }^{\circ}$ 12.187, de 29 de dezembro de 2009. Política Nacional de Mudança Climática, em 30 de novembro de 2009. Diário Oficial [da] República Federativa do Brasil. Brasília, DF, 29 dez. 2009. Seção 1 - Edição Extra, P. 109.

GOLDEMBERG, J. O caminho até Joanesburgo. Meio ambiente no Século 21 (Energia). Rio de Janeiro, p. 171-181. 2003.

HELM, D. Climate-change policy: why has so little been achieved? Oxford Review of Economic Policy, v. 24, n. 2, p. 211-238, 2008

IPEA - INSTITUTO DE PESQUISA ECONÔMICA APLICADA. Perspectivas sobre as negociações de mudança climática e seus impactos na política brasileira. Comunicados do IPEA - $\mathrm{n}^{\circ} 45$. Brasília, 22 de abril de 2010. 15 p.

IPCC - INTERGOVERNMENTAL PANEL ON CLIMATE CHANGE. IPCC special report on carbon dioxide capture and storage. Cambridge: Cambridge University Press, Cambridge. Preparado pelo Grupo de Trabalho III do IPCC. 2005.

IEA - INTERNATIONAL ENERGY AGENCY. Energy technology perspectives 2006: scenarios \& strategies to 2050. Paris: Stedi Media, 2006. Disponível em: < http://www.iea.org/publications/free_new_ Desc.asp?PUBS_ID=2078 >. Acesso em: 23 nov. 2008.

LINO, U.R.A., 2005. Case History of Breaking a Paradigm: Improvement of an Immiscible GasInjection Project in Buracica Field by Water Injection at the Gas/Oil Contact. SPE Latin American and Caribbean Petroleum Engineering Conference, Rio de Janeiro. http://www.spe.org. Acessado em março/2009.

MACHADO, C. X. ; KETZER, J.M. ; MARASCHIN, A. J. ; ROCKETT, G. C. ; SBRISSA, G. F. ; CAPORALE, G. ; ROCHA, L. H. S. ; MACALOS, C. L. ; CENTENO, C. ; CRUZ, A. O. . Construção de um SIG para associação de fontes estacionárias e reservatórios geológicos de CO2 no Brasil. In: Ziliotto, 
M.A.. (Org.). Mudanças Climáticas, Sequestro e Mercado de Carbono no Brasil. 1 ed. Paraná: Instituto Ecoclima/UFPR, 2009, v. , p. 267-279

NETL - NATIONAL ENERGY TECHNOLOGY LABORATORY. Carbon Dioxide Enhanced Oil Recovery - Untapped Domestic Energy Supply and Long Term Carbon Storage Solution. 2010. Disponível em: < http://www.netl.doe.gov/technologies/oil-gas/publications/EP/small_CO2_eor_primer.pdf $>$. Acessado em janeiro/2011.

PACALA, S.; SOCOLOW, R. Stabilization Wedges: Solving the Climate Problem for the Next 50 Years with Current Technologies. Science, v. 305, n. 5686, p. 968-972, 2004.

PETROBRAS, Programa Brasileiro GHG Protocol. Inventário das Emissões de Gases de Efeito Estufa-2008. 20 p., 2009.

PORTER, G.; BROWN, J. W. Global Environmental Politics. $2^{\text {nd }}$ ed. Boulder, Colorado: Westview Press. 1996.

UNFCCC - UN - UNITED NATIONS FRAMWORK CONVENTION ON CLIMATE CHANGE. Views related to carbon dioxide capture and storage in geological formations as a possible mitigation technology - Submission from Parties (Brazilian Submission on the possible inclusion of Carbon Dioxide Capture and Storage in Geological Formation (CCS) in the Clean Development Mechanism). Bonn. 2010.

WORLEYPARSONS. Strategic Analysis of the Global Status of Carbon Capture and Storage. Report 5: Synthesis Report. Final Report (C) 2009 Global CCS Institute. Canberra, Austrália. 2009. 


\title{
CARBON CAPTURE AND STORAGE TECHNOLOGY: WORLD OVERVIEW AND BRAZILIAN SITUATION
}

\begin{abstract}
The scientific analysis and discussion on the effect of anthropogenic emissions of Greenhouse Gases $(G H G)$ emissions and its consequences on climate change had obtained public notoriety in recent decades. The need for global actions designed to combat global warming move both the public and the private sector. The relationship between environment and economy has been a subject of discussion since the first UN Conference on the Human Environment held in Stockholm in 1972. Due to the production / consumption / environment, the private sector is interested in one of the biggest issues of Global Environmental Governance (GAG). As climate change, although the scientific evidence increases, policy responses have had so far little or no impact on the accumulation of emissions. Technology Capture and Storage of Carbon Dioxide $\left(\mathrm{CO}_{2}\right)$ in Geological Reservoirs (CCS) is recognized as a major form of GHG mitigation. To encourage its use in large-scale resources are available from the international market and other voluntary carbon credit. Another incentive mechanism for its use is the market for carbon credits from Clean Development Mechanisms (CDM), however, it is not possible. The World already has a number of institutions carrying out research on this technology. The main objective of this paper is to present an overview of the technology world's geological $\mathrm{CO}_{2}$ storage analyzing the situation of this technology in Brazil. The methodology of this paper is composed of exploratory, descriptive and analytical theme that focuses on the strategies and techniques of qualitative and quantitative research. For the collection and analysis of secondary data, we used traditional research resources. Initially, we reviewed the literature on CCS technology. In addition, we performed analysis of institutional documents such as reports, studies and projects. The primary data were collected through visits to specialists. As a result the article shows the positioning of various countries on the inclusion of CCS under the CDM, specifically the position of Brazil, the current status of research on the structure of CCS technology in Brazil, the main reasons to invest in this technology in Brazil and finally the identification of gaps for establishing an environment more favorable to the field of technology. We conclude that the Government of Brazil has been absent in structuring actions relating to CCS, leaving to the private sector to lead the development and mastery of CCS technology.
\end{abstract}

Keywords: Geological Storage of CO2. CCS. Carbon Credit. CDM. 